\section{P394 BENEFITS OF A VBIC TO BOTH PATIENTS AND THE LOCAL HEALTH ECONOMY}

Sarah White*, Ben Warner, Phil Mairs, Lynn Cope, Karen Lohoar, Walter Melia, Guy Sisson, Chirag Kothari, Siddharth Birdi. Darent Valley Hospital, Dartford And Gravesham Trust, Dartford, UK

\subsection{6/gutjnl-2020-bsgcampus.468}

Introduction Virtual Biologics and Immunosuppressives Clinics (VBICs) assess inflammatory bowel disease (IBD) patients who are on biologics with a view to optimising treatments whilst also delivering cost savings through the withdrawal of high cost drugs. Darent Valley Hospital(DVH) is a district general hospital without an IBD Nurse. A VBIC was set up for the 1st time in July 2019 to assess the 112 patients on infliximab (IFX) to reassess patients' treatment regularly but also with a view to demonstrating how an IBD nurse could be utilised to deliver cost savings.

Methods Patients were assessed in VBIC by 2 IBD consultants, a pharmacy technician and an IBD administrator and categorised into 5 groups based on their recent investigations: to continue biologics, to reassess before offering withdrawal, to switch, to dose-escalate, or dose de-escalate. Letters were written to the patients' consultant advising next management.

Patients were analysed for: the group that they were assigned to, whether next management was performed and the number of patients offered withdrawal. Potential cost savings were estimated on the assumption that patients in the 'reassess before offering withdrawal' category, were likely to be in remission and would agree to withdraw. Costs were based on the price of IFX being $£ 120 / 100 \mathrm{mg}$ vial.

Results 63 patients were analysed $(62 \%$ male, 59\% with Crohn's, $41 \%$ on concomitant therapy, $13 \%$ had previous loss of response to adalimumab). The mean duration patients had been on IFX was 40 months (SD 32, range 1-140 months) and the mean time to their last disease assessment was 19

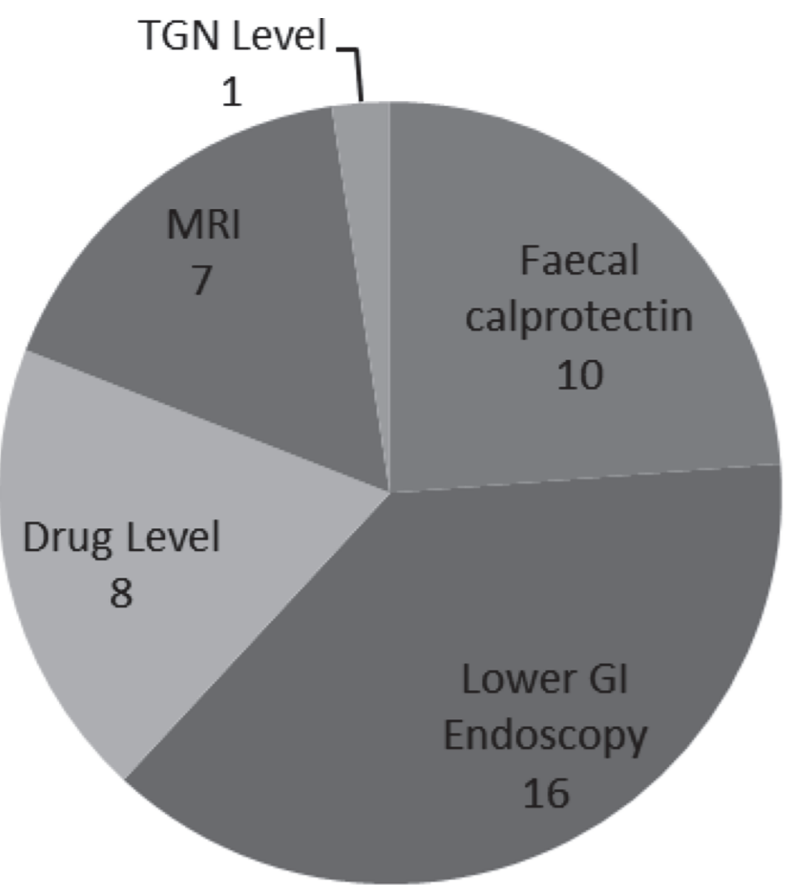

Abstract P394 Figure 1 How patients in the reassess before offering withdrawal category were due to be reassessed months (SD 15, range 1-69 months) of whom 33\% were in remission at that time.

Of the $40(63 \%)$ patients in the 'reassess before offering withdrawal' category only 11 had all the investigations proposed by VBIC performed (see figure 1); only 5(8\%) patients were withdrawn. If all patients in this category had been withdrawn, the savings would have been $£ 162,480$ in the 1 st year and $£ 324,960$ in the 2 nd year.

Of the remaining 23 patients analysed, 3 were switched biologic, 5 were dose-escalated, 1 was de-escalated and 14 continued at their current dose. Overall, 19\% of all the patients had subtherapeutic drug levels.

Conclusions Before VBIC, a significant proportion of our patients had neither been reassessed regularly, nor offered withdrawn when in remission. However, although our VBIC was able to optimise therapy for some patients, it was unable to deliver the predicted cost savings. The reason for this was that it relied on the consultant in charge of the patient's care performing the next management step; an issue that having an IBD nurse would resolve.

\section{Education and training}

\section{P395 UTILISING A DAY-CASE PARACENTESIS UNIT TO DEVELOP A NEAR-PEER TRAINING PROGRAMME FOR JUNIOR DOCTORS}

Rebecca Cliff*, Irene Lee, James Neale. Torbay and South Devon NHS Foundation Trust, UK

\subsection{6/gutjnl-2020-bsgcampus.469}

Introduction Junior doctors traditionally lack confidence in performing diagnostic paracentesis on the medical take. This project explores a near-peer training programme in diagnostic paracentesis, with an aim to improve junior doctors' confidence and subsequent adherence to the national liver care bundle (British Association for the Study of the Liver). It also explores the safety of near-peer training in large volume paracentesis in a day case setting within the gastroenterology team.

Methods A training programme was developed, in which junior doctors interested in learning diagnostic paracentesis were enrolled via email and posters. Training was delivered by four gastroenterology junior doctors, with the consent of patients attending for elective large-volume paracentesis. Trainee survey and clinical audits were used to assess effectiveness and safety of this programme.

Results Feedback showed significant improvement in trainees' confidence in performing the skill. The controlled environment of a day-case setting and interaction with real patients contributed to all trainees finding the setting highly suitable for procedure training. Core trainees also noted that this programme saved them a significant amount of time seeking training opportunities ad-hoc. Re-audit of the liver care bundle showed improvement in the rates of diagnostic paracentesis being performed within 24 hours of admission, from $57.9 \%$ to $65.5 \%$. Over a twelve-month period, gastroenterology junior doctors performed 109 large volume paracenteses. Detailed study of 57 of these cases showed no complications requiring hospital admission.

Conclusion Whilst there may be trends in moving paracentesis towards a specialist nurse-led service with ultrasound guidance, this training programme shows near-peer junior doctor 
teaching of paracentesis remains a viable model for both training and service provision. A controlled day case setting contributes to limiting the influence of human factors on patient safety and complication rates. Furthermore, junior doctors' competence in diagnostic paracentesis remains particularly valuable in out-of-hours acute admission settings. For the future, we believe the training programme has capacity to expand to a wider group of emergency healthcare professionals.

\section{P396 DIFFERENCES IN ENDOSCOPY TRAINING RATES IN WALES - IMPLICATIONS IN THE SHAPE OF TRAINING ERA}

Neil Hawkes, Diluka Karunaratne*, Andrew Phillips. Cwm Taf Morgannwg Uhb, Cardiff, UK

\subsection{6/gutjin-2020-bsgcampus.470}

Background The 'Shape of Training' programme has reduced time for specialty training programmes. Understanding patterns of actual training provision across regions may assist maximising efficient training. For trainees to complete certification before CCST award requires training rates of 50 upper GI points per quarter (200 cases/year) in early training, followed by 50 lower GI points per quarter (100 cases/year).

Aim To provide data on historical patterns of endoscopy training provision for Gastroenterology Specialty Trainees across regional training sites.

Methods Regional data for all Gastroenterology trainees in the Wales deanery from 2013-2019 including procedural data for different endoscopy subtypes and certification information was examined. The numbers of training procedures and procedure points were calculated for all registered trainees at each training site over the study period. To allow comparison across regions a quarterly procedural progression rate (QPPR) was calculated (total training points/number of trainees). Pre- and post-certification procedures were differentiated, trainee periods were out of programme or working LTFT were excluded from analysis.

Results Endoscopic training data on all rotational placements was available for 28 trainees. Training was conducted at 15 sites, in 6 Health Boards (HB) Regions. The table 1 shows QPPR for each region over time. Two-fold differences in training activity for both UGI and LGI endoscopy were seen between the highest and lowest performing HBs. Only 1 out of $6 \mathrm{HBs}$ delivered QPPRs in UGI or LGI endoscopy in the pre-certifcation phase that may be required in the Shape of Training era. Trainees in endoscopy fellowship posts achieved considerably higher PPR (253 for LGI).

Conclusions This study reviewing training provision across a region for seven years revealed differences in provision of training between HBs. Current rates of training delivery would

Abstract P396 Table 1 Quarterly procedural progression rate (QPPR) per HB for UGI and LGI procedures - average points per quarter

\begin{tabular}{lllllll}
\hline & HB1 & HB2 & HB3 & HB4 & HB5 & HB6 \\
\hline UGI Pre & 27.3 & 41.4 & 43.1 & 45.9 & 55.6 & 41.7 \\
UGI Post & 28.5 & 21.5 & 21.2 & 24.9 & 6.8 & 26.0 \\
LGI Pre & 43.3 & 39.9 & 65 & 46.1 & 31.8 & 39.7 \\
LGI Post & 35 & N/A & N/A & N/A & N/A & N/A \\
\hline
\end{tabular}

not allow trainees to achieve full sign off for gastroscopy and colonoscopy within 4 years of specialty training. Formal training fellowships may provide a mechanism to increase PPR significantly.

\section{P397 A PROPOSED EDUCATIONAL PODCAST FOR GASTROENTEROLOGY TRAINEES - HOT AIR OR POTENTIAL HIT?}

${ }^{1}$ Luke Materacki, ${ }^{2}$ James Kennedy*. ${ }^{1}$ Oxford University Hospitals, Oxford, UK; ${ }^{2}$ North Bristol NHS Trust, Bristol, UK

\subsection{6/gutjnl-2020-bsgcampus.471}

Introduction A podcast is a digital audio program, like a radio show, that is available to stream or download. The popularity of podcasts has greatly expanded in recent years and their value is increasingly recognised in modern medical education, particularly in maximising educational opportunities when other learning modalities may be impractical. ${ }^{1}$ The educational appeal of podcasts to the UK gastroenterology trainee is unclear.

This study aimed to explore the attitudes and listening behaviours of UK gastroenterology trainees towards podcasts and investigate appetite for a new podcast with content specifically tailored to their educational needs.

Methods UK gastroenterology trainees were surveyed from 28/ $01 / 2019$ to $10 / 03 / 2019$ using the Google $($ surveys website. The survey was disseminated to trainees nationally through BSG regional trainee representatives using their contact details listed on the BSG website. ${ }^{2}$

Results Of the 42 UK gastroenterology trainees surveyed $78.6 \%$ had listened to a podcast before with $47.6 \%$ having accessed gastroenterology or hepatology content. Several issues with existing podcasts were reported including a paucity of high-quality gastroenterology and hepatology content (80.0\%), poor audio quality $(45.0 \%)$ and content that was either uninteresting $(45.0 \%)$ or inappropriate for the trainee's level of training or understanding (40.0\%). Most trainees had listened to a podcast during a commute to work $(71.4 \%)$ or other destination (38.1\%) and $74.4 \%$ preferred podcasts between 10 and 30 minutes duration.

A high majority of trainees would be interested in listening to a new gastroenterology and hepatology-themed podcast with content specifically tailored to trainees (92.9\%). Desired content included clinical updates (97.4\%), topical items related to gastroenterology or hepatology $(79.5 \%)$ and teaching sessions covering curriculum for the European Specialty Examination in Gastroenterology and Hepatology (74.4\%). Most trainees were unwilling to pay for a new podcast regardless of content $(61.5 \%)$ however $94.9 \%$ would accept a short audio advert to support the cost of podcast production.

Conclusions Although podcast listening is generally popular amongst UK gastroenterology trainees the current experience may be tainted by unsatisfactory content or audio quality. This study uncovered overwhelming appetite for high-quality, short, free-to-access gastroenterology and hepatology-themed podcasts targeting the needs of UK trainees.

\section{REFERENCES}

1. Cho D, Cosimini M, Espinoza J. Podcasting in medical education: a review of the literature. Korean J Med Educ. 2017;29(4):229-239.

2. https://www.bsg.org.uk/resource/trainees-committee.html; accessed 11/02/2019 